Pacific Journal of Mathematics

EMBEDDINGS OF COMPACT CONVEX SETS AND LOCALLY 


\title{
EMBEDDINGS OF COMPACT CONVEX SETS AND LOCALLY COMPACT CONES
}

\author{
JIMMIE D. LAwSON
}

The main result of this paper is that a compact convex set with a basis of neighborhoods (not necessarily open) at each point which are convex can be embedded in a locally convex separated topological vector space. An analogous result is proved for locally compact cones. Along the way it is shown that any compact convex set can be embedded as a base of a locally compact cone in a separated topological vector space, and that the various notions of local convexity coincide in a compact convex set.

1. Introduction. The outstanding open problem in the area with which this paper is concerned has been whether compact convex subsets of general linear spaces can differ from the compact convex subspaces of locally convex spaces or some mild variant thereof. For example, the following is a question posed by V. Klee [8]: Does every element of a compact convex subset $K$ of a topological vector space possess a basis of neighborhoods whose intersection with $K$ is convex?

The main result of this paper provides an affirmative answer for the case that the compact convex set $K$ possesses at each point a basis of neighborhoods (not necessarily open) which are convex. After the original manuscript of this paper'had been written, the author learned that J. W. Roberts had obtained the same result some five or six months earlier, and has submitted it for publication [11]. Roberts has also discovered an example of a compact convex set without extreme points, hence which cannot be embedded in a locally convex space [12]. However, the technique of proof which is employed here is quite different. We use these results to sharpen some earlier results on embedding of cones [9].

The main result is a mild improvement on the work of $R$. E. Jamison, R. C. O'Brien, and P. D. Taylor [5]. In [5], they proved that if a compact, convex set has a base of open convex subsets, then it is affinely homeomorphic to a convex subset of a locally convex topological vector space.

The results for compact convex sets have analogues for locally compact cones; these results are also included. Indeed, the motivation for this paper was a further study of locally compact cones, which arise in a natural way in the theory of compact semigroups [6]. 
The general problem for cones is whether the topology of every locally compact cone $C[\tau]$ in a real vector space $V=C-C$ can be extended to a locally convex separated topological vector space topology $\tau^{*}$ on $V$ such that $\tau^{*} \mid C=\tau$. The finite-dimensional case was disposed of by D. R. Brown and M. Friedberg in [2]. The general problem was first stated and studied by K. Keimel in [7]. B. Madison and I showed that a not necessarily locally convex solution always existed in [9]. This paper gives an affirmative answer if each point of $C$ has a basis of convex neighborhoods in $C[\tau]$, and one may employ the example of Roberts [12] and the construction of $\$ 2$ to show the general answer is no.

2. Embedding compact convex sets into cones. A convex set is defined to be a convex subset of a real vector space. An internal algebraic structure of convex sets can be defined. Such a treatment as well as a proof of the expected theorem on affinely embedding abstract convex sets into linear spaces may be found in $[3,4]$.

A Hausdorff topology on a convex set $X$ such that the mapping $(x, y, t) \rightarrow t x+(1-t) y$ is continuous from $X \times X \times[0,1] \rightarrow X$ is called an affine topology, and $X$ endowed with such a topology is called a topological convex set. Clearly, any convex subset of a linear space (i.e., a topological vector space) is a topological convex set.

Throughout the remainder of the paper, $R$ will denote the real numbers and $H$ the nonnegative real numbers.

A nonempty subset $C$ of a real vector space $V$ is a cone if (i) $x+y \in C$ whenever $x, y \in C$, (ii) $r x \in C$ if $r \in H$ and $x \in C$, (iii) $C \cap-C=\{0\}$. If $C$ is endowed with a Hausdorff topology for which the functions $(x, y) \rightarrow x+y$ from $C \times C$ into $C$ and $(r, x) \rightarrow r x$ from $H \times C$ into $C$ are continuous, then $C$ with this topology is called a topological cone.

A convex subset $B$ of a cone $C$ is an algebraic base for $C$ if for each nonzero element $x$ of $C, H x$ intersected with $B$ is a singleton set (i.e., $B$ meets each ray in a single point). An algebraic base $B$ of a topological cone $C$ is a topological base (or simply a base) if $B$ is a closed subset of $C$.

Proposition 2.1. A compact topological convex set is affinely homeomorphic to a base of a locally compact cone.

Proof. Let $X$ be a compact convex set in some real vector space $V$. Define a new vector space $W=R \times V$, and set

$$
C=\{(t, t v) \in R \times V: 0 \leqq t \text { and } v \in X\} .
$$

It is straightforward to show using the convexity of $X$ that $C$ is a cone 
and $B=\{1\} \times X$ is a base for $C$. Explicitly, the addition in $C$ can be obtained from the convex structure of $X$ by the formula

$$
(t, t v)+(s, s w)=\left(t+s,(t+s)\left(t(t+s)^{-1} v+s(t+s)^{-1} w\right)\right)
$$

provided not both $t$ and $s$ are 0 . This formula may be used to verify that the mapping from $X$ onto $B$ sending $v$ to $(1, v)$ is an affine isomorphism.

Define a basis for a topology on $C$ by taking sets of the form $B(U, V)$ where $U$ is open in $H \backslash\{0\}, V$ is open in $X$, and

$$
N(U, V)=\{(t, t v): t \in U, v \in V\} .
$$

In addition, at $(0,0)$, define a basis of open sets $N_{t}$ for $t>0$ by

$$
N_{t}=\{(s, s v): 0 \leqq s<t\}
$$

It is easily verified that the collection

$$
\{N(U, V): U \text { is open in } H \backslash\{0\}, V \text { is open in } X\} \cup\left\{N_{t}: t>0\right\}
$$

is a basis for a topology $\mathcal{U}$ on $C$.

An alternate description can be given for this topology. Let $\rho: H \times X \rightarrow C$ be defined by $\rho(t, v)=(t, t v)$, and topologize $C$ with the quotient topology. Call this topology $\mathscr{V}$. It follows easily that the inverse image under $\rho$ of any basic open set for $\mathcal{U}$ is open in $H \times$ $X$. Hence, the identity mapping from $(X, \mathscr{V})$ to $(X, \mathcal{U})$ is continuous. Conversely, if $V$ is any open set in $\mathscr{V}$ containing $(t, t x)$, $t \neq 0$, then $\rho^{-1}(V)$ is an open set containing $(t, x)$. Hence there exist open sets $A \subset H, \quad B \subset X$, such that $(t, x) \in A \times B \subset \rho^{-1}(V)$. Then $N(A, B) \subset V$. Finally, let $V$ be an open set $\mathscr{V}$ containing $(0,0)$. Then $\rho^{-1}(V)$ is an open set containing the compact set $\{0\} \times X$. Since $X$ is compact, there exists $t>0$ such that $[0, t) \times X \subset \rho^{-1}(V)$. Then $N_{t} \subset$ $V$. Hence, $\mathcal{u}=\mathscr{V}$.

It follows that the cone $C$ is locally compact since $\rho^{-1}(t, t x)$ is a singleton if $t \neq 0$ and $\rho^{-1}(0,0)=\{0\} \times X$ is compact. (If a compact set in a locally compact Hausdorff space is shrunk to a point, then the quotient is locally compact.)

Next, we show continuity of addition in $C$. Continuity at the pair $((t, t x),(s, s y))$ for $t \neq 0, s \neq 0$, follows in a straightforward fashion from formula $\left(^{*}\right)$ employing continuity of the affine operation in $X$. Continuity at $((0,0),(0,0))$ also is easy since $N_{t / 2}+N_{t / 2} \subset N_{t}$.

To show continuity at $((0,0),(t, t x))$ where $t \neq 0$, let $\left(t_{\alpha}, t_{\alpha} x_{\alpha}\right)$ be a net converging to $(t, t x)$ and $\left(s_{\alpha}, s_{\alpha} y_{\alpha}\right)$ be a net converging to $(0,0)$. To complete the proof, it is sufficient to show $\left(t_{\alpha}+s_{\alpha}, t_{\alpha} x_{\alpha}+s_{\alpha} y_{\alpha}\right)$ clusters to 
$(t, t x)$. By the definition of the topology on $C$, we have $s_{\alpha} \rightarrow 0$ and $t_{\alpha} \rightarrow t$; hence, $s_{\alpha}+t_{\alpha} \rightarrow t$. Also from the definition of the topology on $C$, the net $x_{\alpha}$ converges to $x$. Since $X$ is compact, the net $y_{\alpha}$ clusters to some $y$ in $X$. Hence $t_{\alpha}\left(s_{\alpha}+t_{\alpha}\right)^{-1} x_{\alpha}+s_{\alpha}\left(s_{\alpha}+t_{\alpha}\right)^{-1} y_{\alpha}$ clusters to $t\left(t^{-1}\right) x+$ $0\left(t^{-1}\right) y=x$. It follows from formula $\left({ }^{*}\right)$ that $\left(t_{\alpha}, t_{\alpha} x_{\alpha}\right)+\left(s_{\alpha}, s_{\alpha} y_{\alpha}\right)$ clusters to $(t, t x)$.

Finally, it follows directly from the definition of the topology on $C$ that the affine isomorphism from $X$ into $C$ sending $x$ into $(1, x)$ is a homeomorphism.

We quote now the principal result of [9].

Proposition 2.2. Let $C$ be a locally compact cone. Then the vector space $V=C-C$ may be endowed with a topology for which $V$ is a Hausdorf topological vector space and for which the original topology on $C$ agrees with the relative topology from $V$.

Corollary 2.3. A compact topological convex set is affinely homeomorphic to a base of a locally compact cone in a topological vector space.

A convex set is said to be finite-dimensional if the vector space which it spans is finite-dimensional.

Corollary 2.4. A finite-dimensional compact topological convex set is affinely homeomorphic to a compact, convex subset of $E^{n}$, for some $n$.

Proof. Note that in the proof of Proposition 2.1 if the original space $V$ is finite-dimensional, then also $W=R \times V$ is finitedimensional. Hence the cone $C$ is finite-dimensional, and hence the vector space $C-C$ is finite-dimensional. Hence, this corollary follows from Corollary 2.3, since a finite-dimensional vector space admits only one topology for which it is a separated topological vector space.

We note, in closing this section, that we began by defining topological convex sets in abstract real vector spaces. We concluded by showing that compact topological convex sets may just as well be thought of as compact convex subsets of a Hausdorff topological vector space.

3. Morphisms of convex sets. We now define a category $\mathscr{C}$ of topological convex sets. Objects of $\mathscr{C}$ are just topological convex sets. A morphism between objects $X$ and $Y$ in $\mathscr{C}$ is a continuous function $f$ from $X$ into $Y$ such that if $a, b \in X$ and $0 \leqq t \leqq 1$, then $f(t a+(1-t) b)=t f(a)+(1-t) f(b)$. Morphisms in $\mathscr{C}$ are called affine 
mappings. We define $\mathscr{K}$ to be the full subcategory of $\mathscr{C}$ with objects all compact topological convex sets.

A topological convex set $X$ is said to be strongly locally convex if every compact convex subset of $X$ has a basis of open neighborhoods which are convex.

PROPOSITION 3.1. If a locally compact convex set $X$ has the property that every compact convex set has a basis of convex neighborhoods (not necessarily open), then $X$ is strongly locally convex.

Proof. Let $K$ be a compact convex subset of $X$, and let $U$ be an open set containing $K$. We may assume that $U$ is chosen small enough that $U^{*}$ is compact without loss of generality.

By regularity and the compactness of $K$, there exists an open set $U_{1}$ such that $K \subset U_{1} \subset U_{1}^{*} \subset U$. By hypothesis, there exists a convex neighborhood $W_{1}$ of $K$ such that $W_{1} \subset U_{1}$.

By the same technique, pick a convex neighborhood $W_{2}$ of $W_{1}^{*}$ such that $W_{2}^{*} \subset U$, and continue inductively. Hence, for each $i, W_{i}$ is convex, and $W_{1-1}^{*} \subset$ interior $\left(W_{1}\right) \subset W_{i}^{*} \subset U$. Then $W=\bigcup_{i=1}^{\infty} W_{i}$ is an open convex neighborhood of $K$ contained in $U$.

Proposition 3.2. Let $X$ be a convex subset of a locally convex topological vector space. Then $X$ is strongly locally convex.

Proof. Let $K$ be a compact convex subset of $X$. Then, all $(K+$ $U) \cap X$, where $U$ varies over all convex open neighborhoods of 0 , form a base of open convex neighborhoods of $K$ in $X$.

PROPOSITION 3.3. Let $X$ and $Y$ be compact convex sets and let $f$ be an affine mapping from $X$ onto $Y$. If $X$ is strongly locally convex, then so is $Y$.

Proof. Let $K$ be a compact convex subset of $Y$ and let $U$ be an open set containing $K$. Then, $f^{-1}(K)$ is a compact set in $X$ and $f^{-1}(U)$ is an open set containing $f^{-1}(K)$. Hence, there exists a convex neighborhood $W$ of $f^{-1}(K)$ such that $W \subset f^{-1}(U)$. By compactness $f$ is a closed mapping; hence, $f(W)$ is a (not necessarily open) neighborhood of $K$, and $f(W) \subset U$. By Proposition 3.1, $Y$ is strongly locally convex.

A topological convex set is said to be locally convex if every point has a basis of open convex neighborhoods (Jamison, O'Brien, and Taylor call this concept "strongly locally convex" [5]). Obviously, in our terminology, a convex set is locally convex if it is strongly locally convex.

The principal result of Jamison, O'Brien, and Taylor [5] is the following. 
THEOREM 3.4. Let $X$ be a locally compact topological convex set which is locally convex. Then there is an affine homeomorphism from $X$ onto a convex subset of a locally convex topological vector space.

It follows from this theorem and Proposition 3.2 that for locally compact convex sets, the notions of strong local convexity and local convexity agree.

4. Weakly locally convex sets. This section seems more susceptible to a running account rather than being neatly blocked off into propositions and proofs.

A topological convex set $X$ is said to be weakly locally convex at $p \in X$ if $p$ has a basis of neighborhoods (not necessarily open) in $X$ which are convex. The set $X$ is said to be weakly locally convex if it is weakly locally convex at each point.

Let $C[\tau]$ be a topological cone where $\tau$ is the topology on $C$. Let $E$ be the real vector space $C-C$. Then it is not difficult to verify that all sets of the form $U-U$ where $U$ is chosen from some base of neighborhoods of 0 in $C[\tau]$ form a basis at 0 for a Hausdorff topological vector space topology $\varphi^{*}$ on $E=C-C$ (see $[7,5.4]$ ).

Now assume through the remainder of this section that $C[\tau]$ is locally compact. Then, according to Proposition 2.2, there exists a Hausdorff topological vector space topology $\tau^{*}$ on $E$, which when restricted to $C$, gives $\tau$. Let $V$ be any open neighborhood of 0 in $\tau^{*}$. Then there exists an open set $U, 0 \in U$, such that $U-U \subset V$. Then $(U \cap C)-(U \cap C) \subset$ $U-U \subset V$. Hence the topology $\varphi^{*}$ is finer than the topology $\tau^{*}$. (They, in fact, agree if and only if $E$ is finite-dimensional, but that is not germane to our development.) However, a subset $N$ of $C$ is a neighborhood of 0 in $C[\tau]$ if and only if it is in $C[\varphi]$ (where $\varphi$ is the restriction of $\varphi^{*}$ to $C$ ). (Again, see $[7,5.4]$.)

If $K$ is a compact subset of $C[\tau]$ and $W$ is a neighborhood of 0 in $C[\tau]$, then there exists $r>0$ such that $K \subset r W$. Then $K-K \subset$ $r(W-W)$. Hence $K-K$ is bounded in $E\left[\varphi^{*}\right]$. On the other hand, suppose $A$ is bounded in $E\left[\varphi^{*}\right]$. Then, if $W$ is a compact neighborhood of 0 in $C[\tau]$, there exists $t>0$ such that $A \subset t(W-W)$. Hence the closure of $A$ in $E\left[\tau^{*}\right]$ is compact. Hence a subset $B$ is bounded in $E\left[\varphi^{*}\right]$ if and only if there exists a compact subset $K$ of $C[\tau]$ such that $B \subset K-K$.

Now, assume that $C[\tau]$ is weakly locally convex at 0 . Then $E\left[\varphi^{*}\right]$ has a basis of bounded convex neighborhoods of the form $W-W$ where $W$ is a precompact convex neighborhood of 0 in $C[\tau]$. Hence $E\left[\varphi^{*}\right]$ is normable. Furthermore, it can be shown that $E\left[\varphi^{*}\right]$ is complete and hence a Banach space (see Theorem 5.4 of [7] and the remark afterwards). 
Now, suppose $X$ is a compact topological convex set. By Proposition $2.1, X$ is affinely homeomorphic to a base of a locally compact cone. In fact, if $X$ were a convex subset of a vector space $V$, then the cone in which $X$ was embedded was

$$
C=\{(t, t v) \in R \times V: 0 \leqq t \text { and } v \in X\}
$$

In this cone, a base of neighborhoods of $(0,0)$ were defined for each $t>0$ by

$$
N_{t}=\{(s, s v): 0 \leqq s<t\}
$$

Since each of these sets is easily seen to be convex, $C$ is locally convex at 0 . Hence $X$ is affinely homeomorphic to a base of a locally compact cone which is locally convex at 0 . theorem.

With this preliminary discussion we are now ready for the major

THEOREM 4.1. Let $X$ be a compact topological convex space which is weakly locally convex. Then $X$ is strongly locally convex.

Proof. By the remarks preceding the theorem, $X$ is affinely homeomorphic to a compact base $B$ of a locally compact cone $C[\tau]$ which is locally convex at 0 . If $E=C-C$, then, by the preceding discussion, $E$ admits a topology $\varphi^{*}$ which is a Banach space topology and for which $\varphi$ is finer than $\tau$.

Let $E^{\prime}$ denote the dual of $E\left[\varphi^{*}\right]$ endowed with the norm topology, and let $E^{\prime \prime}$ denote the dual of $E^{\prime}$ endowed with the weak-star topology from $E^{\prime}$. Let $P$ be the closure of $\gamma(B)$ in $E^{\prime \prime}$ (where $\gamma$ is the natural embedding of $E$ into $\left.E^{\prime \prime}\right)$. Then, by Alaoglu's theorem, $P$ is compact (since $B$ is bounded in $E[\varphi]$ and hence in $E^{\prime \prime}$ endowed with the norm topology), convex, and strongly locally convex (since $E^{\prime \prime}$ is locally convex). The set $P$ will play a role for us similar to that which the regular Borel probability measures played in the work of Jamison, O'Brien, and Taylor [5].

We wish now to define a continuous affine function $\sigma$ from $P$ onto $B$.

Let $\gamma\left(x_{\alpha}\right)$ be a net in $\gamma(B)$ converging to a point $p$. Since $B[\tau]$ is compact, the net $x_{\alpha}$ has at least one cluster point $x$ in $B$. Suppose that $y$ is another one, $y \neq x$. Pick closed convex subsets $M$ and $N$ of $B[\tau]$ such that in the $\tau$ topology on $B, x$ is a member of the interior of $M, y$ is a member of the interior of $N$, and $M \cap N=\varnothing$ (this is possible since $B$ is weakly locally convex; this the only point in the proof where this hypothesis is employed). Since $M$ and $N$ are compact, there exists in 
$E\left[\tau^{*}\right]$ an open neighborhood $U$ of 0 such that $(M+U) \cap(N+U)=$ $\varnothing$. Since $\varphi^{*}$ is finer than $\tau^{*}$ and $\varphi^{*}$ is locally convex, there exists an open convex neighborhood $V$ of 0 in $E\left[\varphi^{*}\right]$ such that $V \subset U$. Then $M+V$ and $N+V$ are open, disjoint convex sets in $E\left[\varphi^{*}\right]$.

As a consequence of the Hahn-Banach theorem, there exists an $f \in E^{\prime}$ such that if $a \in M+V$ and $b \in N+V$, then $f(a)<f(b)$. Since $f$ is open, it follows that there exists $\epsilon>0$ such that if $c \in M$ and $d \in N$, then $f(c)+\epsilon<f(d)$.

Now, since $\gamma\left(x_{\alpha}\right)$ converges to $p$ in the weak-star topology on $E^{\prime \prime}$, $\left\langle f, \gamma\left(x_{\alpha}\right)\right\rangle$ converges to $\langle f, p\rangle$. But $\left\langle f, \gamma\left(x_{\alpha}\right)\right\rangle=\left\langle x_{\alpha}, f\right\rangle=f\left(x_{\alpha}\right)$, and since $x_{\alpha}$ is cofinally in both $M$ and $N$, the net $f\left(x_{\alpha}\right)$ cannot be a Cauchy net, and hence cannot converge. This contradiction implies that in $B[\tau]$ the net $x_{\alpha}$ can have at most one cluster point, and hence must converge to a single point $x$.

If $\gamma\left(x_{\beta}\right)$ were another net in $\gamma(B)$ converging to $p$, then also $x_{\beta}$ converges to the same point $x$. For, if not, using the two nets $\gamma\left(x_{\alpha}\right)$ and $\gamma\left(x_{\beta}\right)$, one could construct a new net converging to $p$ for which the inverse image net, with respect to $\gamma$, in $B[\tau]$ clustered to more than one point. But we have just seen this is impossible.

Hence we can define a function $\sigma: P \rightarrow B$ by taking a point $p$ in $P$, any net $\gamma\left(x_{\alpha}\right)$ in $\gamma(B)$ converging to $p$, and declaring $\sigma(p)$ to be the limit of the net $x_{\alpha}$. Employing constant nets, we see $\sigma \mid \gamma(B)=\gamma^{-1}$. Using the uniqueness of limits and the regularity of $B$, it is a straightforward argument to show that $\sigma$ is continuous (see Theorem $1, \S 8.5$, Chapter I of [1]). Since $\sigma=\gamma^{-1}$ on a dense set and $\gamma^{-1}$ is a convex function, the continuity of $\sigma$ implies that it is also a convex function.

Thus, by Proposition 3.3, $B$, and hence $X$, is strongly locally convex.

COROLlaRy 4.2. In a locally compact convex set $X$ the notions of weakly locally convex, locally convex, and strongly locally convex agree. Hence a locally compact convex set which is weakly locally convex can be affinely embedded in a locally convex topological vector space.

Proof. Let $X$ be a locally compact convex set which is weakly locally convex. Let $K$ be a compact convex subset of $X$ and let $U$ be an open set containing $K$. For each $x \in K$, pick a compact convex neighborhood of $x$. Finitely many of these cover, say $V_{1}, \cdots, V_{n}$. Then the convex hull $W$ of $\bigcup_{i=1}^{n} V_{1}$ is also compact since it is the continuous image of the compact set $V_{1} \times \cdots \times V_{n} \times S_{n}$ where

$$
S_{n}=\left\{\left(x_{1}, \cdots, x_{n}\right): 0 \leqq x_{1} \leqq 1 \text { for each } i \text { and } \sum_{i=1}^{n} x_{i}=1\right\} .
$$


Now, $W$ as a compact convex set is also weakly locally convex. Hence, by Theorem $4.1, W$ is strongly locally convex. Pick a convex neighborhood $V$ of $K$ open in $W$ and contained in $U$ intersected with the interior of $W$. Then $V$ is also open in $X$.

The second half of the corollary now follows immediately from Theorem 3.4.

COROLLARY 4.3. Let $C$ be a locally compact cone which is weakly locally convex. Then $C$ is linearly homeomorphic to a locally compact cone in a locally convex topological vector space.

Proof. By Corollary 4.2, there exists an affine homeomorphism $f: C \rightarrow E$ where $E$ is a locally convex topological vector space. Let $p=f(0)$, and define $T: E \rightarrow E$ by $T(x)=x-p$. Then $T \circ f$ is an affine homeomorphism from $C$ into $E$ which carries 0 to 0 . It is not difficult to verify that such a homeomorphism is linear.

5. An alternate approach. At the end of the proof of Corollary 4.2 , it was necessary to rely on the earlier work of Jamison, O'Brien and Taylor [5] in order to complete the proof. If one is willing to assume their results, this provides the quickest route to the desired solution. In this section, we sketch an alternate to the end of the proof of Corollary 4.2 which bypasses the use of their results, is more natural to the development of this paper, and would actually be a more efficient route for someone starting from scratch. This section is essentially an addendum, and the reader interested only in the main results may stop at the end of $\S 4$.

We first need a variant version of Proposition 3.3.

Proposition 5.1. Let $X$ and $Y$ be topological convex sets and let $f$ be an affine mapping from $X$ onto $Y$ which is also a quotient mapping. If $X$ is locally compact, $\sigma$-compact, and strongly locally convex, then $Y$ is locally convex.

Proof. For each $x \in X$, pick a compact convex neighborhood $N_{x}$ of $x$. Since $X$ is $\sigma$-compact, countably many, say $\left\{N_{1}: i \in \omega\right\}$, cover $X$. Let $A_{1}=N_{1}$. Let $B_{2}$ be the convex hull of $A_{1} \cup N_{2}$. Since $B_{2}$ is the continuous image of $A_{1} \times N_{2} \times I, B_{2}$ is compact. Pick $A_{2}$ to be a compact convex neighborhood of $\boldsymbol{B}_{2}$ (using strong local convexity). Continuing by induction, one chooses a compact convex $A_{m}$ for every $m$ such that $N_{m} \cup A_{m-1} \subset A_{m}$ and $A_{m}$ is a neighborhood of $A_{m-1}$. It follows that $X=\bigcup_{m=1}^{\infty} A_{m}$.

Now, let $y \in Y$, and let $U$ be an open set containing $y$. Then $f^{-1}(y)$ is a closed convex subset of $X$ contained in the open set $V=f^{-1}(U)$. By 
renumbering from some $m$ on if necessary, we may assume $f^{-1}(y) \cap$ $A_{1} \neq \varnothing$.

Let $P_{1}=f^{-1}(y) \cap A_{1}$. By strong local convexity, there exists a compact convex neighborhood $W_{1}$ of $P_{1}$ such that $W_{1} \subset V$. Let $Q_{1}=$ $A_{1} \cap W_{1}$. Since $Q_{1}$ is compact and $Y$ is Hausdorff, $f^{-1}\left(f\left(Q_{1}\right)\right)$ is closed. Let $P_{2}=f^{-1}(f(Q)) \cap A_{2} ; P_{2}$ is compact and convex and $P_{2} \subset$ $V$. Let $W_{2}$ be a compact convex neighborhood of $P_{2}$ such that $W_{2} \subset V$ and let $Q_{2}=W_{2} \cap A_{2}$. Since $A_{2}$ is a neighborhood of $A_{1}, Q_{2}$ is a neighborhood of $Q_{1}$ in $X$.

Continuing this process inductively, we get an increasing sequence of compact convex sets $\left\{Q_{n}\right\}_{n \in w}$ such that for each $n$,

(i) $Q_{n+1}$ is a neighborhood of $Q_{n}$,

(ii) $Q_{n+1} \supset f^{-1}\left(f\left(Q_{n}\right)\right) \cap A_{n+1}$,

(iii) $Q_{n} \subset V$, and

(iv) $f^{-1}(y) \cap A_{n} \subset Q_{n}$.

Let $Q=\cup_{n=1}^{\infty} Q_{n}$.

By condition (i), $Q$ is open, by condition (ii), $f^{-1}(f(Q))=Q$, by (iii), $f(Q) \subset U$, and by (iv), $f^{-1}(y) \subset Q$. Since $f$ is a quotient mapping and $f^{-1}(f(Q))=Q$ is open, $f(Q)$ is open. Since each $Q_{n}$ is convex, $Q$ is convex, and hence $f(Q)$ is convex. Hence $y \in f(Q) \subset U$, as desired.

Remark. With only a little more work, it can be shown that $Y$ is actually strongly locally convex. To do this, one must employ the result that $f$ must be a compact-covering mapping; then in the construction, it may be assumed without loss of generality that $f\left(A_{1}\right)$ contains the compact convex set for which a basis of convex neighborhoods is desired. However, the given version is sufficient for our purposes.

Proposition 5.2. If $X$ and $Y$ are strongly locally convex locally compact convex sets, then so is $X \times Y$.

Proof. It will, a priori, be the case that $X$ and $Y$ are locally convex; it follows easily from the definition of the product topology that $X \times Y$ is locally convex. The proposition now follows from Corollary 4.2.

THEOREM 5.3. Let $C[\tau]$ be a locally compact weakly locally convex cone in a real vector space $V=C-C$. If $\eta: C \times C \rightarrow V$ is defined by $\eta(c, d)=c-d$, then $V$ with the quotient topology $\tau^{*}$ is a Hausdorff locally convex topological vector space such that $\tau^{*} \mid C=\tau$.

Proof. Everything but the local convexity of $V$ is proved in Theorem 3.2 of [9] (see also [10] for background and generalizations).

By the first part of Corollary 4.2, $C[\tau]$ is strongly locally convex. Hence, by Proposition 5.2, $C \times C$ is strongly locally 
convex. Thus, by Proposition 5.1, $V$ is locally convex (since locally compact cones are $\sigma$-compact [7]).

Now, if $X$ is a compact weakly locally convex set, then, by the discussion of $\S 4, X$ is affinely homeomorphic to the base of a locally compact cone. If one examines the definition of the topology of this cone in $\$ 2$, then it becomes evident that the cone is also weakly locally convex. Hence, the affine embedding of $X$ into a locally convex separated topological vector space follows from Theorem 5.3. The locally compact case may be obtained from the compact one as in $\S 5$ of [5].

\section{REFERENCES}

1. N. Bourbaki, General Topology, Part I, Addison-Wesley, Readıng, Mass., 1966.

2. D. R. Brown and M. Friedberg, Representation theorems for uniquely divisible semigroups, Duke Math. J., 35 (1968), 341-352.

3. S. Dubuc, Topological convex sets, unpublished dissertation, Cornell University, Ithaca, New York, 1966.

4. S. P. Gudder, Convex structures, Research Publication MS-R 7101, Department of Mathematics, University of Denver, 1970.

5. R. E. Jamison, R. C. O'Brien, and P. D. Taylor, On embedding a compact convex set into a locally convex topological vector space, Pacific J. Math., 64 (1976), 193-205.

6. K. Keimel, Eine Exponentialfunction für kompakte abelische Halbgruppen, Math. Z., 96 (1967), $7-25$.

7. L L L L L Kal kompakte Kegelhalbgruppen und deren Einbettung in topologische Vektorräume, Math. Z., 99 (1967), 405-428.

8. V. L. Klee (Editor), Proc. of the Symposia in Pure Math., Amer. Math. Soc., 7 (1963).

9. J. D. Lawson and B. Madison, On congruences and cones, Math. Z., 120 (1971), 18-24.

10. B. Madison, Congruences in topological semigroups, Proc. of the Second Florida Symposium on Automata and Semigroups, Part II, 1971.

11. J. W. Roberts, The embedding of compact convex sets in locally convex spaces, to appear Canad. J. Math.

12. - A compact convex set with no extreme points, to appear.

Received December 23, 1975 and in revised form August 3, 1976. This research was supported in part by NSF Grant MPS 73-08812.

LOUISIANA STATE UNIVERSITY 



\section{PACIFIC JOURNAL OF MATHEMATICS}

\section{EDITORS}

RICHARI) ARENS (Managing Editor)

University of California

Los Angeles, CA 90024

R. A. Beaumiont

University of Washington

Seattle, WA 98105

\section{J. DugunduI}

Department of Mathematics University of Southern California Los Angeles, CA 90007

D. Gilbarg and J. Milgram Stanford University

Stanford, CA 94305

\section{ASSOCIATE EDITORS}

E. F. BECKENBACH
B. H. NEUMANN

F. Wolf

K. YoshidA

\section{SUPPORTING INSTITUTIONS}

UNIVERSITY OF BRITISH COLUMBIA CALIFORNIA INSTITUTE OF TECHNOLOGY

UNIVERSITY OF CALIFORNIA

MONTANA STATE UNIVERSITY

UNIVERSITY OF NEVADA

NEW MEXICO STATE UNIVERSITY

OREGON STATE UNIVERSITY

UNIVERSITY OF OREGON

OSAKA UNIVERSITY

\author{
UNIVERSITY OF SOUTHERN CALIFORNIA \\ STANFORD UNIVERSITY \\ UNIVERSITY OF HAWAII \\ UNIVERSITY OF TOKYO \\ UNIVERSITY OF UTAH \\ WASHINGTON STATE UNIVERSITY \\ UNIVERSITY OF WASHINGTON \\ AMERICAN MATHEMATICAL SOCIETY
}

The Supporting Institutions listed above contribute to the cost of publication of this Journal, but they are not owners or publishers and have no responsibility for its contents or policies.

Mathematical papers intended for publication in the Pacific Journal of Mathematics should be in typed form or offset-reproduced (not dittoed), double spaced with large margins. Underline Greek letters in red, German in green, and script in blue. The first $p: 1<$ graph or two must be capable of being used separately as a synopsis of the entire paper. Items of the biblography should not be cited there unless absolutely necessary, in which case they must he identified by author and Journal, rather than by item number. Manuscripts, in duplicate, may be sent to any one of the four editors. Please classify according to the scheme of Math. Reviews, Index to Vol. 39. All other communications should be addressed to the managing editor, or Elaine Barth, University of California, Los Angeles, California, 90024.

100 reprints are provided free for each article, only if page charges have been substantially paid. Additional copies may be obtained at cost in multiples of 50 .

The Pacific Journal of Mathematics is issued monthly as of January 1966. Regular subscription rate: $\$ 72.00$ a year (6 Vols., 12 issues). Special rate: $\$ 36.00$ a year to individual members of supporting institutions.

Subscriptions, orders for back numbers, and changes of address should be sent to Pacific Journal of Mathematics, 103 Highland Boulevard, Berkeley, California, 94708.

PUBLISHED BY PACIFIC JOURNAL OF MATHEMATICS, A NON-PROFIT CORPORATION Printed at Jerusalem Academic Press, POB 2390, Jerusalem, Israel.

\section{Copyright (C) 1976 Pacific Journal of Mathematics} All Rights Reserved 


\section{Pacific Journal of Mathematics}

\section{Vol. 66, No. 2 December, 1976}

Gerald A. Beer, Tax structures whose progressivity is inflation neutral..... 305

William M. Cornette, A generalization of the unit interval............. 313

David E. Evans, Unbounded completely positive linear maps on

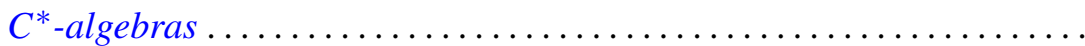

Hector O. Fattorini, Some remarks on convolution equations for

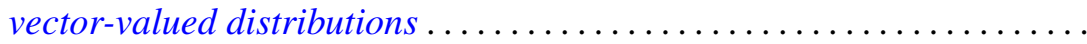

Amassa Courtney Fauntleroy, Automorphism groups of unipotent groups of

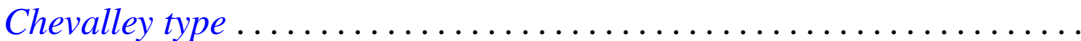

Christian C. Fenske and Heinz-Otto Peitgen, On fixed points of zero index in

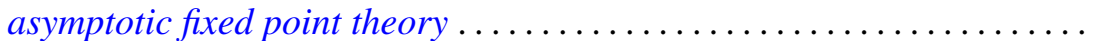

Atsushi Inoue, On a class of unbounded operator algebras. II ............

Herbert Meyer Kamowitz, The spectra of endomorphisms of algebras of

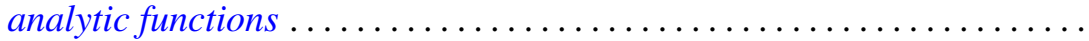

Jimmie Don Lawson, Embeddings of compact convex sets and locally compact cones ....................................

William Lindgren and Peter Joseph Nyikos, Spaces with bases satisfying certain order and intersection properties .....................

Emily Mann Peck, Lattice projections on continuous function spaces ...... 477

Morris Marden and Peter A. McCoy, Level sets of polynomials in $n$ real variables...

Francis Joseph Narcowich, An imbedding theorem for indeterminate Hermitian moment sequences......................

John Dacey O'Neill, Rings whose additive subgroups are subrings ...

Chull Park and David Lee Skoug, Wiener integrals over the sets bounded by sectionally continuous barriers .....................

Vladimir Scheffer, Partial regularity of solutions to the Navier-Stokes equations.

Eugene Spiegel and Allan Trojan, On semi-simple group algebras. II

Katsuo Takano, On Cameron and Storvick's operator valued function space integral 